\title{
Globalbudget - Bundesrat überschreitet rote Linie
}

\section{Michael Kohlbacher}

Dr. iur., MPH, Generalsekretär

Der Bundesrat macht Ernst mit seiner Kostendämpfungsstrategie im KVG. Er schlägt die Umsetzung von ersten Massnahmen in allen grossen Kostenblöcken ("Spital stationär», "Arztbehandlungen ambulant», "Arzneimittel», "Spital ambulant») vor, setzt aber schwergewichtig im ambulanten Bereich an [1]. Die Massnahmen führen zu einem ambulanten Globalbudget und in Verbindung mit der Verlagerung stationärer Leistungen in den ambulanten Sektor zu Rationierungen ambulanter Leistungen. Damit überschreitet der Bundesrat eine rote Linie - aus Sicht der ambulant tätigen Ärzteschaft, aber vor allem aus Sicht der Patientenschaft, die auf eine gute Gesundheitsversorgung vertraut und diese auch will.

\section{Das Gesundheitssystem bietet beste Versorgung für alle}

Das Schweizer Gesundheitssystem schützt seine Bevölkerung im internationalen Vergleich am besten vor vorzeitiger Sterblichkeit, die durch den Indikator «Vorzeitige vom Gesundheitsversorgungssystem potentiell beeinflussbare Sterblichkeit (Mortality Amenable to Health Care, MAHC)" gemessen wird. In die Berechnung fliessen Todesursachen ein, die unmittelbar durch das Gesundheitsversorgungssystem beeinflussbar gewesen wären, und "vorzeitig» bezieht sich auf ein Alter von unter 75 Jahren. In der Schweiz gibt es pro 100000 Einwohner nur 41 Fälle von vorzeitiger Sterblichkeit, die bei rechtzeitiger und effektiver Gesundheitsversorgung nicht eingetreten wären. Das ist im OECD-Vergleich der absolut tiefste Wert [2].

Der Gesundheitszustand der verschiedenen sozioökonomischen Gruppen in der Schweiz unterscheidet sich weniger stark als in anderen Ländern, was nicht zuletzt auf einen umfassenden Zugang der Bevölkerung zu den Gesundheitsdienstleistungen zurückzuführen ist. In anderen europäischen Ländern, in denen Kostendämpfungs- und Rationierungsmassnahmen ergriffen

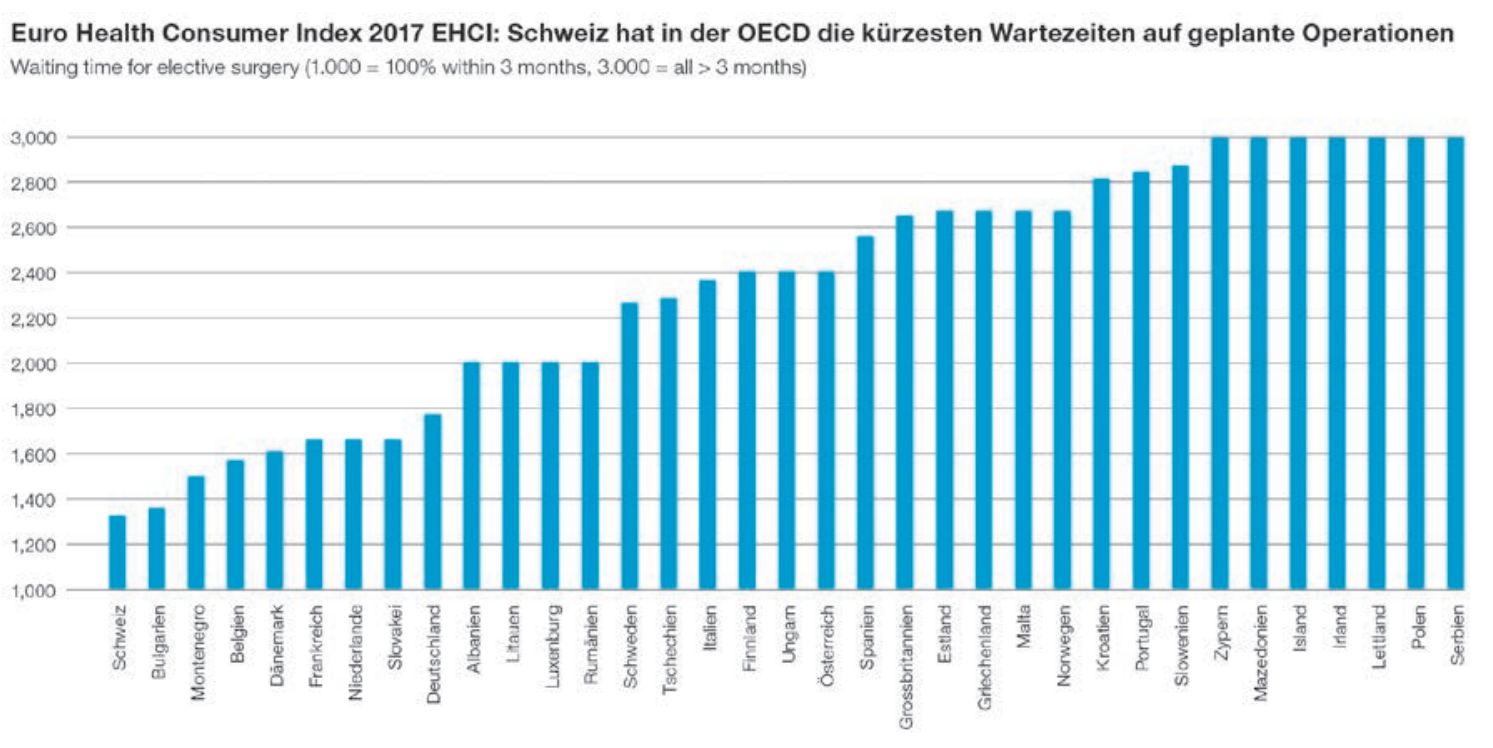


wurden, sind bspw. zum Teil hohe Wartezeiten auf «Wahleingriffe», d.h. Operationen in nicht unmittelbar lebensbedrohlichen Situationen, zu verzeichnen. Gemäss Euro Health Consumer Index 2017 hat die Schweiz in der OECD die kürzesten Wartezeiten für koronare Bypass-Operationen/PTCA (Herzkranzgefässerweiterungen) und Hüft- und Knieoperationen (Abb 1.) [3]. Diese Indikatoren zeigen auf, dass das Schweizer Gesundheitssystem im Zuge der Entwicklungen der letzten Jahrzehnte eine Versorgungsqualität für die Gesamtbevölkerung, unabhängig von Alter und sozioökonomischem Status, erreicht hat, die in anderen Ländern, die der Bundesrat zurzeit beispielgebend für seine Kostendämpfungsstrategien zitiert, nicht vorhanden ist.

\section{Das hohe Versorgungsniveau ist finanzierbar}

Ungeachtet aller Unkenrufe muss bei einer sachlichen Betrachtung festgestellt werden, dass die Schweizer Gesundheitsversorgung zurzeit finanziell tragbar ist und auch in der Zukunft noch finanziell tragbar wäre, wenn für Gesundheitsausgaben bspw. 15\% des Bundesinlandprodukts verwendet würden. Es ist auch nicht so, dass das KVG, trotz der Einführung zahlreicher zusätzlicher Leistungen, der starken Bevölkerungszunahme und der demographischen Alterung, keinen Kostendämpfungseffekt gehabt hätte. Der inflationsbereinigte reale jährliche Kostenanstieg betrug in 20 Jahren KVG 3,5\%, im Vergleich zu 3,7\% vor der Einführung des KVG [4].

Die Haushaltsbudgeterhebungen des Bundesamtes für Statistik zeigen, dass sich die Budgetsituation der Haushalte durch die steigenden Gesundheitskosten und Krankenkassenprämien nicht verschlechtert hat: Den Haushalten stand im Durchschnitt nach Abzug von Steuern, Krankenkassenprämien und sonstigen Sozialabgaben im Jahr 2015 sogar um 14\% mehr frei verfügbares Einkommen zur Verfügung als im Jahr 2008 [5].

\section{Finanzierungslast besser verteilen}

Wenn auch bei einer Durchschnittsbetrachtungsweise die Bevölkerung kein Finanzierungsproblem hat, so belasten die hohen Krankenkassenprämien vor allem zunehmend den Mittelstand. Die Krankenkassenprämien dürfen aber im Kontext der Sozialausgaben nicht für sich allein betrachtet werden. Die Fiskalquote, die die effektiven Steuereinnahmen und Sozialversicherungsabgaben im Verhältnis zum nominalen Bruttoinlandprodukt (BIP) darstellt, ist in der Schweiz im internationalen Vergleich relativ tief: Mit 27,8\% ist sie annähernd gleich hoch wie in den USA (26\%) und liegt deutlich un- ter dem OECD-Durchschnitt (34,3\%); in anderen europäischen Ländern beträgt sie zum Teil über 40\% [6]. Es erscheint aus dieser Perspektive überraschend, warum in der Schweiz als einem Land mit einer im internationalen Vergleich tiefen Steuerbelastung gerade jetzt die steuerfinanzierten Prämienverbilligungen zurückgehen und damit die Prämienbelastung für Versicherte mit tieferen Einkommen erhöht wird. Von einer gesundheitspolitischen Strategie des Bundesrats und der Kantone könnten zugunsten der Aufrechterhaltung einer hochstehenden Gesundheitsversorgung für alle Einkommensklassen Vorschläge im Hinblick auf eine bessere Verteilung der Finanzierungslast, insbesondere durch einen Ausgleich aus Steuermitteln, erwartet werden.

\section{Der Nutzen der Gesundheitsversorgung wird verschwiegen}

Man sollte meinen, dass die Kostendämpfungsmassnahmen vom Bundesrat vorgeschlagen werden, weil der Nutzen der Gesundheitsversorgung den Kosten nachhinkt und die Bevölkerung nicht angemessen von den Leistungen des Schweizer Spitzengesundheitssystems profitiert. Demgegenüber zeigen Umfragen, dass die Bevölkerung mit dem Status quo der Gesundheitsversorgung sehr zufrieden ist und keinen Abbau von Leistungen, sondern die Beibehaltung des hohen Niveaus der Leistungen aus der Grundversicherung wünscht. Die Zahlungsbereitschaft der Bevölkerung, um den hohen Nutzen der Gesundheitsversorgung erhalten zu können, ist sehr hoch. Die Bereitschaft, für eine Kostensenkung individuelle Einschränkungen hinzunehmen, geht sogar tendenziell zurück [7].

Es ist unverständlich, warum sich der Bundesrat bei seinem Vorschlag von Kostendämpfungsmassnahmen nicht damit auseinandersetzt, mit welchem Nutzenverlust für die Bevölkerung diese Vorschläge einhergehen. Es wird im Gegenteil mit der Fokussierung der Kostendämpfungsmassnahmen auf den ambulanten Sektor der Hebel dort angesetzt, wo die Bevölkerung den grössten Nutzen sieht. Eine Einschränkung der freien Arztwahl, sei es nach Kosten-, Qualitäts- oder Effizienzkriterien, sieht die Bevölkerung als den grössten Nutzenverlust an, respektive müssten die Krankenkassenprämien dafür sehr stark sinken, um den Nutzenverlust in Kauf zu nehmen (Abb. 2) [8].

\section{Ambulant vor stationär + Deckelung ambulant $=$ Rationierung}

Die staatlich verordnete Ökonomisierung der Spitalversorgung durch das DRG-System hat keine Einsparungen gebracht und zur Verschiebung von Leistungen und Kos- 
Nutzenverluste von Reformvarianten pro Versicherten und Monat (2004)

Die Schweizer Bevölkerung wünscht eine umfassende Grundversicherung für alle, bei Leistungskürzungen müssten die Prämien stark sinken (Studienergebnisse Polynomics: H. Telser, Wie viel ist uns die Qualität im Gesundheitswesen wert? Die Volkswirtschaft 3/2017)

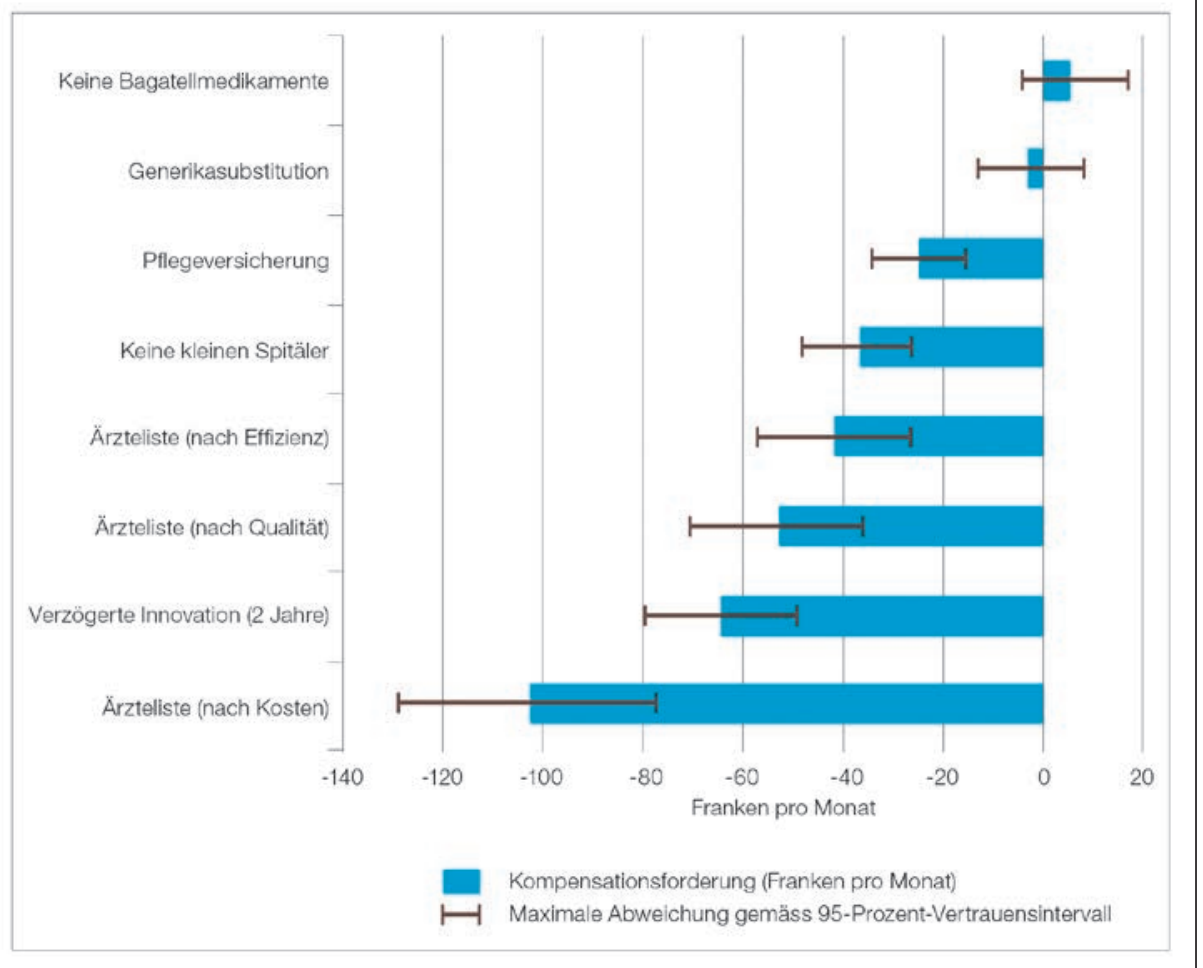

ten geführt, indem vorher in Spitälern erbrachte, von den Tagespauschalen abgegoltene Leistungen in den ambulanten Sektor ausgelagert wurden. Ist das alte System ein Anreiz für zu lange Spitalaufenthalte, stellt das neue System einen Anreiz zur Mengenausweitung von stationären und ambulanten Leistungen in Spitälern und von ambulanten Leistungen ausserhalb von Spitälern dar. Eine weitere (zu begrüssende) Verlagerung von stationären Leistungen in den ambulanten Sektor findet zurzeit durch nationale bzw. kantonale Listen von vorwiegend ambulant zu erbringenden Leistungen statt. Mitten in diese Verlagerungsstrategien von Leistungen in den ambulanten Sektor hinein bezweckt nun der Bundesrat, insbesondere mit den Steuerungsmassnahmen der ambulanten Kosten gemäss Art. 47c KVG, die ambulanten Leistungen zu deckeln und ein Globalbudget einzuführen. Es gibt aber keinen Überlaufkanal mehr. Anders als bei der Verlagerung von stationär zu ambulant kann eine vom ambulanten Sektor nicht mehr erbringbare Leistung nicht durch eine andere Leistung substituiert werden. Das bedeutet zwangsweise eine Rationierung von ambulanten Gesundheitsleistungen.

\section{Kostendämpfungsstrategie im Blindflug}

Diese Strategie verfolgt der Bundesrat ohne finanzielle Not, ohne Datengrundlage und ohne «Health Impact Assessment»: Die Gesetzesvorlage ist schon betreffend finanzielle Auswirkungen ein Blindflug, denn nicht einmal darüber ist in den Materialien etwas nachzulesen. Noch viel weniger beschäftigt sich der Bundesrat damit, mit welchen Auswirkungen auf die Gesundheitsversorgung der Bevölkerung die Deckelung ambulanter Leistungen einhergeht. Dass die unweigerlich drohenden Rationierungen ambulanter Leistungen negative Auswirkungen auf die Gesundheit der Bevölkerung haben werden, liegt auf der Hand. Der schwarze Peter dafür wird den Leistungserbringern zugeschoben, denen unter dem Versorgungsdruck zugemutet wird, Leistungen zu nicht kostendeckenden Tarifen oder sogar auf unbezahlte Weise zu erbringen.

Es ist eine Paralyse der Gesundheitspolitik festzustellen, die sich seit Jahren in einer nahezu irrationalen Weise exklusiv mit mehr oder weniger untauglichen Mitteln zur Kostendämpfung beschäftigt, entgegen der Faktenlage die Finanzierbarkeit der Gesundheitsversorgung in Frage stellt und konsequent verweigert, sich mit dem Nutzen einer guten Gesundheitsversorgung für alle auseinanderzusetzen.

\section{Forderungen der AGZ an Gesundheits- reformen des Bundesrats}

Die AGZ hat in einer Stellungnahme zur Gesetzesvorlage den Bundesrat aufgefordert, die Teilrevision des KVG mittels der vorliegenden unreflektierten Steue- 
rungsmassnahmen der ambulanten Kosten zurückzuziehen. Der Bundesrat soll die ihm seit 2015 auch für den ambulanten Sektor vorliegenden Strukturdaten zur Hand nehmen und zusammen mit anderen Daten in ein umfassendes Assessment der Leistungsfähigkeit, der Kosten-Nutzen-Situation, der volkswirtschaftlichen Finanzierbarkeit, von Finanzierungslösungen und von Effizienzpotentialen des Schweizer Gesundheitsversorgungssystems mit einbeziehen. Danach sollen neue Vorschläge gemacht werden, deren erstes Ziel die Aufrechterhaltung des Leistungsniveaus und des Nutzens und deren nachgelagertes Ziel die Finanzierbarkeit sein soll. Die Leistungserbringer, die in der aktuellen Strategie ohne Gehör mit Kostendämpfungsstrategien bedient werden, sollen in diese Analyse und Ausarbeitung von Lösungsvorschlägen mit einbezogen werden.

\section{Richtiger Ansatz für Gesundheitsreformen}

Das Gesundheitsversorgungssystem und seine Reformierung sollten sich zuerst am Bedarf und den Bedürfnissen der Menschen orientieren, die die Versorgungsleistungen benötigen. Das Gesundheitsversorgungssystem und seine Reformierung sollten zweitens die Voraussetzungen dafür schaffen, dass die Angehörigen der Gesundheitsberufe die benötigten Leistungen unter guten Bedingungen und mit guter Qualität erbringen können. Das Gesundheitsversorgungssystem und seine Reformierung sollten drittens dafür sorgen, dass die Finanzierung dieser Leistungen in einer angemessenen, von der Gesamtwirtschaft und den Menschen tragbaren Höhe und Form sichergestellt ist.

Wie oben dargelegt, geht der Bundesrat in seiner Gesundheitsreform anders vor. Er stützt sich auf technokratische Analysen von Experten, die zudem wenig fundiert sind, nicht das halten dürften, was sie versprechen und Kollateralschäden für Leistungsempfänger und Leistungserbringer verursachen werden.

\section{Angebot der AGZ - Beitrag der Zürcher Ärzteschaft für Gesundheitsreformen}

Die AGZ beschränkt sich nicht darauf, die Gesundheitsreformvorschläge des Bundesrats zu kritisieren. Die AGZ ist bereit, einen eigenen Beitrag zu leisten und aufzuzeigen, wie eine Gesundheitsreform anders aufgegleist werden könnte.

Der Vorstand der AGZ hat ein anerkanntes Forschungsinstitut beauftragt, mit einer qualitativen und quantitativen Analyse den Bedarf und die Bedürfnisse der Menschen, die das Versorgungssystem potentiell oder tatsächlich in Anspruch nehmen, und der Menschen, die Versorgungsleistungen anbieten, zu erheben. Erhoben wer- den nicht nur die Ansprüche an die Versorgung bzw. an die Rahmenbedingungen für die Erbringung der Versorgungsleistungen, sondern auch Erwartungen betreffend Versorgungsstrukturen sowie Finanzierungsaspekte. In einem ersten Schritt wird vom Forschungsinstitut im Rahmen von Fokusgruppeninterviews die Sichtweise der "Leistungsempfänger», differenziert nach jungen Menschen, Menschen mittleren Alters, betagten Menschen und Menschen mit chronischen Krankheiten, und der «Leistungserbringer» (Ärzte), differenziert nach zukünftigen Ärzten (Studierende sowie Ärzte in Weiterbildung) und Ärzten im Berufsalltag, erhoben. In einem zweiten Schritt wird aufgrund der Erkenntnisse der Fokusgruppeninterviews eine repräsentative quantitative Erhebung durchgeführt werden. Die Ergebnisse dieser Studie werden im April 2019 vorliegen. Die AGZ wird somit im Frühjahr 2019 aufzeigen können, wie das Versorgungssystem aus Sicht der Betroffenen aussehen sollte.

\section{Referendum gegen unbegründete Rationierung und Leistungsabbau}

Mit diesem Vorgehen zeigt die AGZ beispielgebend auf, dass die Ärzteschaft gewillt und bereit ist, eine gute Gesundheitsversorgung aktiv mitzugestalten. Für einen unbegründeten Leistungsabbau, der die Gesundheitsversorgung der Bevölkerung verschlechtert, wird die AGZ nicht die Hand bieten. Die AGZ hat daher in ihrer Stellungnahme dem Bundesrat offen mitgeteilt, dass sie für den Fall, dass das Gesetzesvorhaben mit der Deckelung der ambulanten Kosten in dieser Form durchgezogen werden soll, das Referendum ergreifen bzw. ein von der Ärzteschaft ergriffenes Referendum mittragen wird.

Bildnachweise

Abbildung 1: Euro Health Consumer Index 2017. https://healthpower house.com/media/EHCI-2017/EHCI-2017-report.pdf

Abbildung 2: Telser H. Die Volkswirtschaft 3/2017. http://dievolkswirt schaft.ch/content/uploads/2017/02/DV 3 2017_DE.pdf

Literatur

1 Vernehmlassungsunterlagen auf Website des BAG: https://www. bag.admin.ch/bag/de/home/versicherungen/krankenversiche rung/krankenversicherung-revisionsprojekte/kvg-revisionmassnahmen-zur-kostendaempfung-Paket-1.html

2 Outcome-Messung im Gesundheitswesen. Bundesministerium für Arbeit, Soziales, Gesundheit und Konsumentenschutz. Wien 2018, S. 59

3 Euro Health Consumer Index 2017. https://healthpowerhouse. com/media/EHCI-2017/EHCI-2017-report.pdf, S. 69.

4 Froidevaux A, Kilchenmann C. Ein statistischer Rückblick auf 20 Jahre KVG. Soziale Sicherheit 3/2016.

5 Tabellen der Haushaltsbudgeterhebungen auf Website des BFS: https://www.bfs.admin.ch/bfs/de/home/statistiken/ wirtschaftliche-soziale-situation-bevoelkerung/einkommen verbrauch-vermoegen/haushaltsbudget

6 Eidgenössische Finanzverwaltung EFV. Fiskal- und Steuerquote der Schweiz im internationalen Vergleich. 19.6.2018.

7 gfs Gesundheitsmonitor 2018.

8 Telser $\mathrm{H}$. Wie viel ist uns die Qualität im Gesundheitswesen wert? Die Volkswirtschaft 3/2017, S. 23-6. http://dievolkswirtschaft.ch/ content/uploads/2017/02/DV_3_2017_DE.pdf 